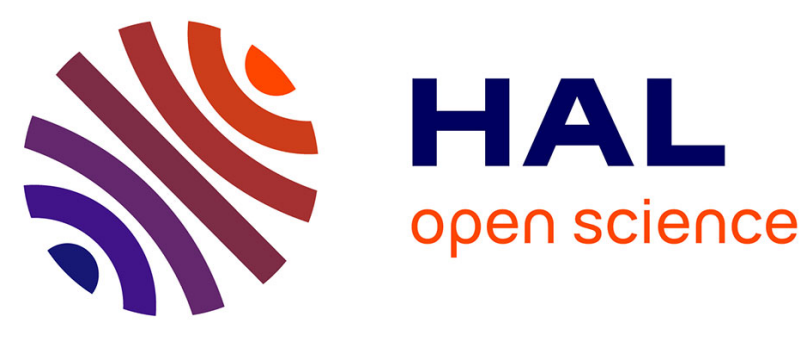

\title{
Théories sans frontières ?
}

Lucile Dumont

\section{To cite this version:}

Lucile Dumont. Théories sans frontières ?: Les théories littéraires en France et la construction d'un espace transnational, années 1960-années 1970.. Actes de la Recherche en Sciences Sociales, 2018, 224 (4), pp.46-63. 10.3917/arss.224.0046 . halshs-01910688

\section{HAL Id: halshs-01910688 https://shs.hal.science/halshs-01910688}

Submitted on 12 Nov 2018

HAL is a multi-disciplinary open access archive for the deposit and dissemination of scientific research documents, whether they are published or not. The documents may come from teaching and research institutions in France or abroad, or from public or private research centers.
L'archive ouverte pluridisciplinaire HAL, est destinée au dépôt et à la diffusion de documents scientifiques de niveau recherche, publiés ou non, émanant des établissements d'enseignement et de recherche français ou étrangers, des laboratoires publics ou privés. 


\section{THÉORIES SANS FRONTIÈRES ?}

Les théories littéraires en France et la construction d'un espace transnational, années 1960-années 1970

Lucile Dumont

Le Seuil | «Actes de la recherche en sciences sociales »

$2018 / 4 \mathrm{~N}^{\circ} 224$ | pages 46 à 63

ISSN 0335-5322

ISBN 9782021401127

Article disponible en ligne à l'adresse :

https://www.cairn.info/revue-actes-de-la-recherche-en-sciencessociales-2018-4-page-46.htm

\section{Pour citer cet article :}

Lucile Dumont, « Théories sans frontières ? Les théories littéraires en France et la construction d'un espace transnational, années 1960-années 1970 », Actes de la recherche en sciences sociales 2018/4 ( $\left.\mathrm{N}^{\circ} 224\right)$, p. 46-63.

DOI 10.3917/arss.224.0046

Distribution électronique Cairn.info pour Le Seuil.

(C) Le Seuil. Tous droits réservés pour tous pays.

La reproduction ou représentation de cet article, notamment par photocopie, n'est autorisée que dans les limites des conditions générales d'utilisation du site ou, le cas échéant, des conditions générales de la licence souscrite par votre établissement. Toute autre reproduction ou représentation, en tout ou partie, sous quelque forme et de quelque manière que ce soit, est interdite sauf accord préalable et écrit de l'éditeur, en dehors des cas prévus par la législation en vigueur en France. Il est précisé que son stockage dans une base de données est également interdit. 


\section{POWTIQUE \\ Revue de théorie et d'analyse littéraires \\ Publiée avec le concours du Service des Publications de \\ Paris-Sorbonne}

Comité de Rédaction : Hélène Cixous, Gérard Genette

27 rue Jacob

Voici le premier numéro d'une revue qui se propose de promouvoirfà la fois dans le champ universitaire et dans l'ensemble de ce public qui,en lisant, s'intez roge sur ce qu'est en elle-même la littératureponton la connaissance de la littérarité. Projet dans lequel la littérature ne peut être séparée de toutes ces manifestations plus ou moins proches que sont le folklore, les communications de masse, etc. Projet qu'une équipe de jeunes chercheurs français entend mener en collaboration étrojte avec les travaux effectués sur tous ces thèmes, aujourd'hui, aussi bien à l'étranger.

\section{Somaire du № 1}

\section{Présentation}

- Roland Barthes : Par où commencer? glúc.

- Jean-Pierre Richard: Balzac, de la force à la forme. metho de Muiuetiqu.

- Harald Weinrich : Structures narratives du mythe

- Hélène Cixous : I'écriture comme placement (Henry James) $\psi$ a ualy tipue

- Philippe Lacoue-Labarthe : La fable (Littérature et philosophied mithede

- Christiane Veschambre : Lecture des "Impressions d'Afrique" Raverite founel.

\section{Mise au point}

( - Hans Robert Jauss : Littérature médiévale et théorie des genres

Document

- Tzvetan Todorov : Le nombre, la lettre, Ie mot

- Vélémir Khlebnikov : iivre les préceotes (I) 


\title{
Lucile Dumont
}

\section{Théories sans frontières?}

\author{
Les théories littéraires en France et la construction \\ d'un espace transnational, années 1960-années 1970
}

Les méthodes d'analyse de la littérature développées en France dans les années 1960 et 1970 et fortement inspirées de la linguistique structurale ont connu un succès rapide, et rapidement international. En témoignent notamment les établissements prestigieux qui les ont adoptées en France et à l'étranger au nom de la « French Theory », ou les critiques et autres canulars dont certains de leurs auteurs ont fait l'objet ${ }^{1}$. Pourtant, ces méthodes ne rassemblent pas une école, un groupe ni un corpus de méthodes unifiées. Leur hétérogénéité et l'instabilité des labels qui les désignent sont caractéristiques des luttes qui animent les champs littéraire, académique et intellectuel $^{2}$. Régulièrement désignées sous le nom de « théories littéraires » ou « théorie de la littérature », ces méthodes ne sont toutefois pas uniformément revendiquées comme telles. Lorsqu'elles le sont, c'est principalement dans trois directions. La première d'entre elles correspond à la revendication de méthodes scientifiques ${ }^{3}$, susceptibles de rompre avec l'empirisme naif et les approches dites « externes » et positivistes de la littérature, alors dominantes dans les études littéraires et l'enseignement supérieur : les analyses psychologiques et biographiques, la philologie, l'histoire littéraire ${ }^{4}$ en particulier. Deuxièmement, la revendication du label de « théorie littéraire » dans les années 1960, dans le cadre de la diffusion du structuralisme et alors que le marxisme s'impose dans les sciences humaines, devient un marqueur politique ${ }^{5}$ qui fait de la théorie l'instrument privilégié de la critique des idéologies en littérature - le petit groupe de la revue Tel Quel en étant l'un des foyers les plus visibles à partir de la fin des années 1960. Troisièmement, l'idée de théorie renvoie, et ce bien au-delà de la période étudiée ici, à la volonté de construire ce que Gérard Genette a défini comme une «théorie générale des formes littéraires $»^{6}$, c'est-à-dire un ensemble d'outils analytiques spécifiques à la littérature, qui soient transhistoriques et, dans la mesure du possible, transnationaux. Il ne s'agit ainsi plus de penser la production littéraire à partir de son contexte de production, donc à travers des catégories qui ne lui sont pas spécifiques - historiques, psychologiques, géographiques par exemple - mais à partir de l'étude « interne » et « immanente », de ce qui est défini comme le fonctionnement propre à la littérature, sa « littérarité ». L'usage de la théorie est donc également revendiqué comme un moyen d'affirmer l'autonomie des études littéraires par rapport aux autres disciplines des sciences humaines.

Ces trois usages du label de théorie littéraire, non exclusifs, se retrouvent à différentes régions de la « nébuleuse $»^{7}$ des promoteurs des approches théoriques de la littérature, ensemble diffus et très hétérogène dont l'unité toute relative est peut-être à trouver dans l'opposition au modèle scolaire de l'histoire littéraire attribué à l'héritage universitaire de Gustave Lanson. En effet,
1. Voir par exemple Alan Sokal et Jean Bricmont, Impostures intellectuelles, Paris, Odile Jacob, 1997.

2. Voir Anna Boschetti, Ismes. Du réalisme au postmodernisme, Paris, CNRSÉd., 2014. 3. Sur ces questions, je me permets de renvoyer à : Lucile Dumont, "Faire théorie pour faire science? Modèles scientifiques et production théorique dans les études littéraires en France (1960-1972) ", Revue d'histoire des sciences humaines, 31, 2017, p. 17-42.

4. Voir Antoine Compagnon, La Troisième République des lettres, de Flaubert à Proust, Paris, Seuil, 1983.

5. Voir Frédérique Matonti, « La politisation du structuralisme. Une crise dans la théorie ", Raisons politiques, 18, 2005, p. 49-71 et, "L'anneau de Mœbius. La réception en France des formalistes russes ", Actes de la recherche en sciences sociales, 176-177, 2009, p. 52-67.

6. Gérard Genette, Figures III, Paris, Seuil, 1972, p. 10.
7. Voir Christian Topalov (dir.), Laboratoires du nouveau siècle. La nébuleuse réformatrice et ses réseaux en France, 1880-1914, Paris, Éd. de l'EHESS, 1999 et Vincent Kaufmann, La Faute à Mallarmé. L'aventure de la théorie littéraire, Paris, Seuil, 2011. 
sous l'appellation de « théorie littéraire » s'opposent de nombreux sous-groupes, dont les labels indigènes changent de sens dans le temps et l'espace. Ainsi la " nouvelle critique », terme qui désigne en France la première génération de promoteurs d'approches théoriques se revendique bien peu de son pendant anglo-américain de New Criticism, dont les approches formalistes et internalistes sont revendiquées par les « nouveaux critiques » français sur le tard, alors même qu'aux États-Unis les tenants du New Criticism sont pour certains farouchement opposés à leur déclassement symbolique par l'importation de cette « nouvelle critique ». La sémiotique, sur laquelle l'espace transnational des théories littéraires prend institutionnellement appui, préexiste aussi aux approches théoriques de la littérature développées avec le structuralisme. En France et aux États-Unis, elle est investie par les tenants de la linguistique structurale puis par les promoteurs des théories littéraires, qui transforment la discipline et l'éloignent de sa tradition anglo-américaine. De la même manière, le renouvellement des méthodes d'analyse de la littérature initié en France autour de la revue Poétique est un réinvestissement d'une discipline antique et d'une pensée critique développée par Paul Valéry, toutes deux remaniées par l'appropriation de méthodes structurales. Enfin, et comme le montrent ces oppositions et ces chevauchements, non seulement les labels font l'objet de luttes symboliques, mais ils n'ont cours que périodiquement dans les espaces intellectuels, académiques et littéraires : nécessaires en tant que modalité d'accumulation primitive du capital symbolique, ils sont rapidement dévalués par la logique temporelle des rapports de force dans ces espaces et/ ou abandonnés au profit d'une quête de la singularité pour accéder à la consécration individuelle ${ }^{8}$. C'est ce qui conduit par exemple Tzvetan Todorov à faire changer le titre d'un chapitre pour sa republication en volume, initialement intitulé « le structuralisme en poétique » en Poétique, ce dernier titre lui apparaissant " plus rentable ${ }^{9}$ sur le marché du livre et des biens symboliques au début des années 1970.

La concurrence entre groupes intellectuels, la nécessité de se distinguer, les chassés-croisés temporels et transnationaux dans la circulation des textes et des idées font des théories littéraires un objet difficile à saisir. Pour cette enquête ${ }^{10}$, on a donc tenté de repérer la production théorique qui se développe autour de deux de ses « producteurs cardinaux ${ }^{11}$, Roland Barthes (19151980) et son élève Gérard Genette (1930-2018), figures centrales de deux générations successives de producteurs théoriques respectivement liées à l'émergence de la « nouvelle critique » et à la revue Poétique créée en 1970 par Gérard Genette, Tzvetan Todorov et Hélène Cixous aux éditions du Seuil. Leur inscription dans des réseaux d'agents et d'institutions construit progressivement un espace transnational des théories littéraires, comme en atteste l'apparition d'instances spécifiques à la production théorique ${ }^{12}$. Dans la série de colloques, les revues et l'association internationale qui participent de ces instances, les productions françaises jouent en effet un rôle central et conservent leur position dominante. En plus de l'étude des textes théoriques, nécessaire à la compréhension de leur diffusion, cette enquête s'est déployée sur trois terrains, au moyen d'archives et d'entretiens : un terrain institutionnel (établissements d'enseignement supérieur français et américains), un terrain éditorial (revues, collections éditoriales et traductions) et la reconstitution de trajectoires biographiques de producteurs théoriques.

Au sein des espaces académiques, intellectuels et littéraires français, l'émergence des théories littéraires prend place dans un contexte d'importantes transformations de l'enseignement supérieur, en particulier dans les sciences humaines et sociales ${ }^{13}$. Elle contribue à la professionnalisation de la critique littéraire, renforce la recherche en littérature et renouvelle la discipline des lettres modernes alors en forte croissance ${ }^{14}$. Énoncée en termes théoriques, l'opposition des promoteurs d'approches théoriques aux études littéraires dominantes peut être saisie comme une retraduction de la polarisation du champ académique français dans les années 1960-1970, entre un pôle plus «national », dans lequel se trouvent les études littéraires universitaires, et un pôle plus « international», où se trouvent les sciences sociales et de nouvelles disciplines et au sein duquel se développent les théories littéraires ${ }^{15}$. Le succès rencontré par les approches théoriques de la littérature dans l'enseignement supérieur français et nord-américain est le produit d'une triple logique, nationale (la production théorique, revendiquée comme telle, émerge et se diffuse
8. Voir Gisèle Sapiro et Lucile Dumont, « La diffusion internationale du structuralisme entre appropriation et rejet ", in Jean-Francois Bert et Jérôme Lamy (dir.), Résonances des structuralismes, Paris, Éd. des archives contemporaines, 2016, p. 123-138.

9. Lettre manuscrite de Tzvetan Todorov, datée du 2 septembre 1973, Archives de I'IMEC, SEL 3984.8, dossier "Todorov Fichier analyse ».
10. Cet article est issu d'une thèse de doctorat, provisoirement intitulée "La légitimation d'une avant-garde ? La circulation internationale et la réception des théories littéraires dans l'enseignement supérieur français (années 1960-1980) ».

11. Howard S. Becker, Les Mondes de l'art, Paris, Flammarion, 1988.

12. Voir Gisèle Sapiro, "Le champ est-i national ? La théorie de la différenciation sociale au prisme de l'histoire globale ", Actes de la recherche en sciences sociales, 200, 2013, p. 70-85 et en particulier p. 84. 13. Voir par exemple Antoine Prost et Jean-Richard Cytermann, "Une histoire en chiffres de l'enseignement supérieur en France ", Le mouvement social, 233, 2010, p. 31-46 et, Mathieu Hauchecorne, "Essor et disciplinarisation des sciences humaines et sociales ", in Christophe Charle et Laurent Jeanpierre (dir.), La Vie intellectuelle en France. II. De 1914 à nos jours, Paris, Seuil, 2016, p. 565-589.

14. Voir Clémence Cardon-Quint, Des lettres au français. Une discipline à l'heure de la démocratisation (1945-1981), Rennes, PUR, 2015.

15. Voir Pierre Bourdieu, Homo academicus, Paris, Minuit, 1984, p. 140 sq. 
en premier lieu dans les champs académique, intellectuel et littéraire français), internationale (ce succès repose sur des relations entre des institutions étrangères en grande partie dépositaires de politiques culturelles et pédagogiques nationales) et transnationale, puisque les « rapports existants entre les ressortissants des différents pays » et surtout le « rôle que jouent les États, les entreprises et les institutions internationales dans l'établissement de ces rapports $»^{16}$ incitent à envisager la diffusion des corpus théoriques au-delà de ses représentations officielles. Cependant, et bien qu'il ait été montré que les productions théoriques circulent plus que les productions empiriques ${ }^{17}$, la circulation transnationale des idées dans le domaine littéraire ne va pas de soi : les outils et les méthodes d'analyse de la littérature sont inséparables des textes littéraires auxquels ils sont appliqués. Or ceux-ci sont mobilisés Paul de Manière privilégiée pour la construction d'identités nationales, et leur diffusion est en grande partie dépendante d'instances pédagogiques vouées à la diffusion de cultures nationales ${ }^{18}$. C'est d'ailleurs contre cette logique que la littérature comparée s'est employée depuis le milieu du $\mathrm{XX}^{\mathrm{e}}$ siècle à « situer un lieu disciplinaire indépendant de toute définition nationale ${ }^{19} »$.

Les circulations transnationales, tout en faisant l'objet d'un investissement stratégique de la part des théoriciens, contribuent ainsi Paul de Manière paradoxale à la formation d'une «tradition nationale $»^{20}$ dans les études littéraires en même temps qu'elles apparaissent comme un processus fondamental de construction d'un espace transnational des théories littéraires, hiérarchisé et polarisé. Dès lors, afin de comprendre sous quelles conditions ces théories littéraires entendent devenir, selon le mot du premier numéro de Poétique, des théories « sans frontières $~^{21}$, il faut peut-être moins observer les étapes de la construction d'un espace transnational des théories littéraires que la manière dont « ces savoirs se forgent dans le contexte même de leur "circulation", en contact direct et continu avec leurs usagers, de sorte qu'on ne sait plus qui des formulations savantes ou des usages $[\ldots]$ a précédé l'autre $»^{22}$. Les trajectoires biographiques des promoteurs des approches théoriques et l'usage stratégique qu'ils font des circulations internationales d'une part, de même que la structuration progressive d'un espace transnational au sein duquel la « littérarité » des textes est érigée en valeur commune d'autre part, soulignent ainsi, s'il le fallait encore, la nécessité de réinscrire les productions intellectuelles dans leurs conditions sociales de production, et ce d'autant plus que leur vocation « théorique » les arrache en apparence à ces dernières.

\section{Controverse nationale et reconnaissance internationale}

Lémergence de la " nouvelle critique » renvoie à un épisode fondateur dans la construction des approches théoriques de la littérature et à l'une des figures centrales de leur première génération. Il s'agit de Roland Barthes, chef de travaux à la $6^{e}$ section de l'École pratique des hautes études (EPHE), où il est élu directeur d'études en 1962 sur un poste de « Sociologie des signes, symboles et représentations ». C'est par l'intermédiaire d'une série d'articles ${ }^{23}$ initialement publiés à l'étranger entre la fin des années 1950 et le début des années 1960 qu'il prend position dans la critique littéraire française, illustrant la manière dont la «nouvelle critique » se constitue selon des logiques propres aux espaces nationaux mais en leur opposant d'emblée des formes de reconnaissance internationale. Ces articles sont republiés en France en 1964 dans un recueil, Essais critiques, qui paraît aux éditions du Seuil un an après un autre court recueil monographique de Roland Barthes chez le même éditeur, Sur Racine. Cette série de publications déclenche la controverse avec Raymond Picard (1917-1975), spécialiste de Racine et professeur de littérature française du XVII ${ }^{e}$ siècle à la Sorbonne. Raymond Picard répond à Roland Barthes dans la presse puis dans un court volume publié en 1965, intitulé Nouvelle Critique ou nouvelle imposture ${ }^{24}$. Dans cette « controverse instituante $»^{25}$, les catégories nationales et étrangères sont mobilisées « en tant
16. Abram de Swaan, "Pour une sociologie de la société transnationale ", Revue de synthèse, 119(1), 1998, p. 89-111 et en particulier p. 95

17. Voir Gisèle Sapiro (dir), Sciences humaines en traduction. Les livres français aux États-Unis, au Royaume Uni et en Argentine, Paris, Centre européen de sociologie et de science politique (CESSP)/Institut français, 2014.

18. Voir Anne-Marie Thiesse, "Littérature et éducation au national ", Le français aujourd'hui, 167, 2009, p. 19-26.

19. Emily Apter, "Translatio globale : l'invention de la littérature comparée, Istanbul
1933 ", Littérature, 144, 2006, p. 25-55 et en particulier p. 28

20. Johan Heilbron, "Qu'est-ce qu'une tradition nationale en sciences sociales ? ", Revue d'histoire des sciences humaines, 18, 2008, p. 3-16.

21. "Présentation ", Poétique, 1, 1970

p. 2.

22. Antoine Vauchez, "Le prisme circulatoire. Retour sur un leitmotiv académique ", Critique internationale, 59, 2013, p. 9-16 et en particulier p. 13.

23. Notamment, Roland Barthes, OEuvres complètes, 5 vols, Paris, Seuil, 2002.

R. Barthes, "Voies nouvelles de la critique littéraire en France ", CEuvres complètes I, Paris, Seuil, 2002 [1959], p. 977-980 R. Barthes, "Les deux critiques ", OEuvres complètes II, Paris, Seuil, 2002 [1963] p. 469-501 ; R. Barthes, «Qu'est-ce que la critique? ", CEuvres complètes II, op. cit., p. $502-507$

24. Raymond Picard, Nouvelle Critique ou nouvelle imposture, Paris, Jean-Jacques Pauvert éd., 1965.

25. Jean-Louis Fabiani, "Disputes, polémiques et controverses dans les mondes intellectuels. Vers une sociologie historique des formes de débat agonistiques ", Mil neuf cent. Revue d'histoire intellectuelle,
25, 2007, p. 45-60. L'épisode a fait l'objet d'analyses sur lesquelles je ne reviens pas ici pour des questions évidentes de place. Voir notamment : P. Bourdieu, Homo academicus, op. cit., p. 149 sq. ; Christophe Prochasson, "Les espaces de la controverse. Roland Barthes contre Raymond Picard : un prélude à Mai 68 ", Mil neuf cent. Revue d'histoire intellectuelle, 25, 2007, p. 141155 ; Tiphaine Samoyault, Roland Barthes, Paris, Seuil, 2015, p. 397 sq. ; et le cours d'Antoine Compagnon au Collège de France en février 2011, "Barthes versus Picard ". 
qu'armes » et « en tant qu'enjeux $»^{26}$. La controverse s'inscrit dans un espace transnational déjà balisé par la circulation du paradigme structuraliste, dominée par les pays qui occupent un rôle central sur le marché de la traduction et/ou dans le champ académique international en sciences humaines depuis le début des années 1960 - soit la France, le Royaume-Uni et, progressivement, les États-Unis. Le structuralisme, en tant que paradigme transnational, pose en effet les premiers jalons de l'espace plus spécialisé sur le domaine littéraire qui se dessine dans la décennie suivante. Dans ce contexte, la question du cadrage national ou transnational des approches théoriques de la littérature n'attend pas la traduction et la publication d'ouvrages théoriques à l'étranger pour être posée. Elle apparaît au contraire dès les premières tentatives de définition de la «nouvelle critique » dans des débats en France. Dans la série d'articles qui précède la controverse, Roland Barthes s'oppose à celle qu'il désigne comme la «critique universitaire $»^{27}$. Sous des labels mouvants - « critique de structure » ou « critique fonctionnelle $»^{28}$, puis « critique d'interprétation ou idéologique $»^{29}-$ il rassemble un petit groupe d'auteurs qu'il finit par classer selon quatre « grandes philosophies » (l'existentialisme, le marxisme, la psychanalyse et le structuralisme). Celles-ci rassemblent un groupe hétérogène d'auteurs : Jean-Paul Sartre temporairement, mais surtout Lucien Goldmann, Mauron, René Girard, Jean Starobinski, Georges Poulet et Jean-Pierre Richard, dont il rattache les travaux à ceux de Gaston Bachelard. À propos du structuralisme, qu'il identifie au formalisme et cite parmi les méthodes fécondes, Roland Barthes mentionne Claude LéviStrauss, Ferdinand de Saussure et Roman Jakobson comme fondateurs ${ }^{30}$.

La polarisation occasionnée par la querelle dans les champs académique, intellectuel et littéraire français circonscrit la nébuleuse des théories littéraires et son caractère transnational. Elle illustre la lutte pour le monopole interprétatif des canons littéraires nationaux et a plusieurs conséquences. Premièrement, elle place Roland Barthes à la tête de ce qui est peu à peu envisagé comme une « avant-garde » de la critique littéraire ${ }^{31}$.
Deuxièmement, sa réception internationale « marque $»^{32}$ définitivement la nouvelle critique. Plusieurs articles sont publiés à son sujet en Italie, où le structuralisme connaît très tôt une réception favorable dans les études littéraires ${ }^{33}$. C'est également le cas en Angleterre dans le Times Literary Supplement qui avait publié un texte de Roland Barthes impliqué dans la controverse ${ }^{34}$. Des revues prestigieuses y font référence, dans des articles qui introduisent Roland Barthes aux États-Unis, et ne tardent pas à se l'approprier dans les luttes symboliques locales. Par exemple, en 1969, Stephen Nichols, ancien élève du théoricien de la littérature tchèque-américain René Wellek à Yale, consacre la moitié de son compte rendu de la traduction du Degré zéro de l'écriture en anglais à la controverse, qu'il interprète comme l'opposition des « scientifiques littéraires »(literary scientists) et des «néo-sartriens »(neosartrians) ${ }^{35}$ en prenant clairement parti pour Roland Barthes, qu'il identifie comme le plus radical des premiers. Toujours aux États-Unis, Jean Seznec, ancien directeur du Département des langues et littératures romanes de l'Université Harvard puis titulaire de la chaire de littérature française de l'Université Oxford au Royaume-Uni, fait un compte rendu du petit ouvrage de Raymond Picard dans la revue French Studies en s'attardant sur l' « arrogance », les « aberrations » et les « exemples bouffons » de Roland Barthes, et s'il reconnaît l'intérêt de son Michelet (1954), c'est pour mieux prendre la défense de l'histoire littéraire ${ }^{36}$. Les Quatre conférences sur la «nouvelle critique », tenues en Italie en 1967 et publiées en $1968^{37}$, et le Panorama de la nouvelle critique en France de Robert Emmet Jones, professeur au Massachusetts Institute of Technology (MIT), publié la même année ${ }^{38}$ contribuent à la circulation transnationale de la «nouvelle critique » et de la controverse dans un même mouvement. Henri Peyre, professeur de littérature française à l'Université de Yale, animateur des Entretiens de Pontigny franco-américains ${ }^{39}$ et ancien président de la prestigieuse Modern Language Association (MLA), fait quant à lui référence à certains travaux de la « nouvelle critique » dans sa préface à un recueil de textes de Gustave Lanson publié en $1965^{40}$.
26. Pierre Bourdieu, "Les conditions sociales de la circulation internationale des idées ", Actes de la recherche en sciences sociales, 145, 2002, p. 3-8.

27. R. Barthes, "Les deux critiques", op. cit.

28. R. Barthes, "Voies nouvelles..." op. cit.

29. R. Barthes, "Les deux critiques", op. cit.

30. R. Barthes, "Qu'est-ce que la critique ", op. cit.

31. Voir par exemple Raymond Picard

"M. Barthes et la critique universitaire ",
Le Monde, 14 février 1964.

32. Pierre Bourdieu, "Les conditions sociales de la circulation internationale des idées ", Actes de la recherche en sciences sociales, 145,2002 , p. 3-8 et en particulier p. 8.

33. G. Sapiro et L. Dumont, op. cit.

34. Voir par exemple N. S., "Crisis in criticism: the Picard-Barthes debate", The Times Literary Supplement, 5, 1966, p. 163-171 ; N.S., "Civil war among the critics", The Times Literary Supplement, 3, 1966.

35. Voir par exemple Stephen G. Nichols, Jr., "Review: Roland Barthes", Contemporary
Literature, 10(1), 1969, p. 136-146.

36. Jean Seznec, "Nouvelle critique ou nouvelle imposture, par Raymond Picard ", French Studies, 21(2), 1967, p. 180-181. 37. "Quatre conférences sur la "nouvelle critique". Textes de Franco Simone, Georges Poulet, Jean Starobinski, Kurt Wais et René Girard ", Studi francesi, supplément, 34, 1968.

38. Robert Emmet Jones, Panorama de la nouvelle critique en France. De Gaston Bachelard à Jean-Paul Weber, Paris, SEDES, 1968.

39. Voir Laurent Jeanpierre, « Pontigny-en
Amérique ", in Centre culturel internationa de Cerisy (CCIC), S.I.E.C.L.E., Colloque de Cerisy, 100 Ans de rencontres intellectuelles de Pontigny à Cerisy, Paris, IMEC, 2005, p. 137-154 et, François Chaubet et Emmanuelle Loyer, "L'école libre des hautes études de New York : exil et résistance intellectuelle (1942-1946) ", Revue historique, 616, 2000, p. 939-972.

40. Gustave Lanson, Essais de méthode, de critique et d'histoire littéraire. Textes rassemblés et présentés par Henri Peyre, Paris, Hachette, 1965 
La dimension transnationale de la «nouvelle critique » était par ailleurs présente dans la stratégie initiale de Roland Barthes. Ses trois articles critiques envers la " critique universitaire » avaient en effet été publiés à l'étranger, en partie grâce à la mobilisation de ses ressources et de ses réseaux d'interconnaissance transnationaux, en particulier au Royaume-Uni et aux États-Unis. Le premier article était une commande de Politika, un quotidien de référence de la Yougoslavie de Tito $^{41}$; le deuxième avait paru dans Times Literary Supplement, prestigieux magazine de critique littéraire publié à Londres ; et le troisième dans Modern Language Notes, une importante revue académique consacrée aux littératures européennes liée à l'Université Johns Hopkins à Baltimore aux États-Unis. Roland Barthes considérait que la «nouvelle critique » était « nationale » parce que, selon lui, elle ne devait que « très peu, sinon rien, à la critique anglo-saxonne, au Léo Spitzerisme, au crocisme $»^{42}$. Raymond Picard n'avait pourtant pas manqué de remarquer l'enjeu du positionnement de Roland Barthes dans un espace intellectuel qui dépassait les frontières nationales, en disant sa crainte qu' « auprès d'un public mal informé, cette diffamation futile et irresponsable [ait] plus de chances de réussir $»^{43}$ et son regret de n'être pas parvenu à placer son article de réponse à Roland Barthes paru dans Le Monde dans la sélection hebdomadaire du journal publiée à l'étranger $^{44}$. Troisième conséquence, comme l'écrit Jean Starobinski en 1970, " pour convaincre, pour être suivis, les principaux intervenants [de la controverse] ont dû accentuer le trait $»^{45}:$ la controverse et sa médiatisation radicalisent les positionnements de deux camps qui ne tardent pas à se dessiner et à se politiser. Roland Barthes précise ses conceptions de la critique littéraire dans un volume qui clôt les échanges, Critique et vérité, publié en 1966. Il y fait des opposants à la « nouvelle critique » un « parti conservateur » empreint de nationalisme. Contre les accusations faites à la nouvelle critique d'employer trop de « jargons », dont il rappelle l'antisémitisme de la comparaison de ces derniers à du yiddish par un opposant, Roland Barthes réprouve l'injonction à écrire selon une « clarté » qu'il renvoie au mythe de la « clarté française » en fustigeant les croyances en la supériorité de la langue et de la littérature françaises, qu'il qualifie de «maladie nationale » de l'« ablutionnisme du langage $»^{46}$. L'émergence de la «nouvelle critique » par le biais d'une controverse nationale immédiatement positionnée dans un espace transnational fait apparaître des caractéristiques propres aux logiques d'internationalisation des champs académiques et intellectuels. Celles-ci s'articulent ici à la position dominante du français et de la littérature française sur la scène internationale, qui permet à la critique littéraire française de circuler favorablement à l'étranger. Pour les promoteurs des approches théoriques, le « recours » à l'international apparaît dès lors comme une voie alternative d'accumulation de capital symbolique et une manière de s'opposer au pôle temporellement dominant du champ académique duquel ils sont exclus ${ }^{47}$.

\section{Les espaces internationalisés et les trajectoires transnationales de la « nouvelle critique »}

Les individus ponctuellement rassemblés sous la bannière de la «nouvelle critique " partagent des propriétés sociales et des profils de carrière similaires. À l'exception de Charles Mauron, l'aîné des critiques cités par Roland Barthes et inventeur de la psychocritique, qui décède trois ans après avoir reçu un doctorat de lettres de l'Université de Paris en 1966 et ne participe donc pas au développement des approches théoriques, aucun des «nouveaux critiques » n'est en poste dans des filières littéraires universitaires en France. Ce sont des hommes, nés hors de Paris ou à l'étranger entre le début du XX siècle et les années 1920. Parmi eux, les représentants de l'École de Genève désignés par Roland Barthes forment un premier groupe dont les carrières sont internationales et se déploient spécialement dans des espaces anglophones.

Fils d'un industriel belge, Georges Poulet (19021991) naît près de Liège, où il obtient un doctorat en 1927. Il enseigne d'abord en Écosse à Édimbourg, avant d'être nommé à l'Université Johns Hopkins en 1952. Il y rejoint le critique autrichien exilé Léo Spitzer. Né en 1922 à Marseille, Jean-Pierre Richard entre à l'ENS Ulm en 1941 et obtient l'agrégation de lettres en 1944. Il enseigne à l'étranger (Édimbourg, où il est invité par Georges Poulet, Londres, où il croise Raymond Picard à l'Institut français, Madrid, Naples) jusqu'en 1969, date de son recrutement comme enseignant au Centre universitaire expérimental de Vincennes. Il termine sa carrière à l'Université de la Sorbonne (Paris IV). Les Études sur le temps humain de
2017, p. 190-208.

42. R. Barthes, "Qu'est-ce que la critique? ", op. cit., p. 503.

43. R. Picard, "M. Barthes... ", art. cit.

44. R. Picard, Nouvelle critique ou nouvelle imposture, op. cit., p. 84.
45. Jean Starobinski, La Relation critique, Paris, Gallimard, 1970, p. 11.

46. R. Barthes, "Critique et vérité ", CEuvres complètes II, op. cit., p. 771.

47. Voir Blaise Wilfert, "Cosmopolis et l'homme invisible. Les importateurs de litté- rature étrangère en France, 1885-1914 ", Actes de la recherche en sciences sociales, 144,2002 , p. 33-46. 
Georges Poulet, dont les travaux sur Marcel Proust ont fait date dans la critique, s'appuient sur une réflexion sur la conscience littéraire et marquent son rejet du formalisme. Le travail de Jean-Pierre Richard envisage la littérature dans sa dimension expérimentale et sensible. Avec Jean Starobinski, Georges Poulet et Jean-Pierre Richard forment le noyau de ce qui est parfois désigné comme l'École de Genève, un courant critique qui adapte notamment certains aspects de la phénoménologie à l'analyse des textes littéraires. Jean Starobinski naît en 1920 à Genève, fils de parents médecins (le père est psychiatre), tous deux juifs d'origine polonaise. Il fait une licence de lettres à Genève, qu'il termine en 1942 avant de se tourner vers la médecine, et d'obtenir son doctorat en 1958. Invité par Georges Poulet à Johns Hopkins de 1953 à 1956, il y enseigne comme instructor puis comme assistant professor. Il poursuit sa carrière entre les Universités de Bâle et de Genève, où il enseigne la littérature française et l'histoire des idées. Ses travaux sur la mélancolie et sur le regard en littérature le rapprochent, avec Georges Poulet et Jean-Pierre Richard, de la critique thématique. Enfin, René Girard (1923-2015), originaire d'Avignon, fils d'un conservateur de bibliothèque et de musée et d'une mère bibliothécaire, était diplômé de l'École des Chartes et de l'Université d'Indiana aux États-Unis (1950). Nommé professeur titulaire à Johns Hopkins en 1961, René Girard a effectué la totalité de sa carrière aux États-Unis : il a également enseigné dans les Universités Duke, Bryn Mawr, SUNY Buffalo, Stanford. La littérature n'a pas été son unique objet de recherche, et il est plus connu aujourd'hui pour ses travaux d'anthropologie sur la violence. La publication de Mensonge romantique et vérité romanesque en 1961 puis ses recherches sur Fiodor Dostoïevski l'ont pourtant fait connaitre des littéraires américains, comme en témoigne le prix du MLA qui lui fut attribué en 1965. Il a été coopté à l'Académie française en 2005.

Lobtention de postes à l'étranger, temporaires ou permanents et en particulier dans des espaces totalement ou partiellement anglophones (Royaume-Uni, États-Unis, Suisse) et des établissements prestigieux, et du même coup la formation et/ou la consolidation d'un réseau international d'interconnaissances apparaissent comme des facteurs déterminants dans la constitution de cet espace transnational. L'organisation de colloques internationaux, lieux privilégiés d'échange mais aussi de luttes symboliques à la fois entre pairs appartenant au même champ national et entre pairs issus de champs nationaux différents, participe également de ce processus ${ }^{48}$. En 1966, deux colloques sont ainsi organisés de part et d'autre de l'Atlantique. Le premier, organisé par Georges Poulet ${ }^{49}$, se déroule dans l'été à Cerisy-la-Salle. Il est intitulé «Les tendances actuelles de la critique ». René Girard y prend part. Roland Barthes et Lucien Goldmann en sont absents, mais ils y sont représentés par leurs élèves, respectivement Gérard Genette et Jacques Leenhardt. Sont également présents Serge Doubrovsky, qui publie deux ans plus tard Pourquoi la Nouvelle Critique ${ }^{50}$, Marcel Raymond et Jean Rousset, tous deux rattachés à l'École de Genève, Jean Ricardou, spécialiste du Nouveau Roman et proche de la revue Tel Quel, Paul de Man, un critique d'origine belge établi aux États-Unis et qui y deviendra un des promoteurs de la déconstruction. La « nouvelle critique » est régulièrement abordée dans les discussions de la décade. Sur la vingtaine de conférenciers, au moins neuf sont en poste à l'étranger, dont cinq dans des pays anglophones, conséquence possible des affinités particulières de Cerisy avec le monde anglo-américain. Une seule présentation est spécifiquement consacrée à la critique littéraire à l'étranger, "Aspects de la critique en Italie », par Aldo Rossi. Au mois d'octobre 1966, c'est cette fois René Girard qui organise, avec Richard Macksey et Eugenio Donato à Johns Hopkins, le colloque The languages of criticism and the sciences of man ${ }^{51}$, parfois qualifié de moment de naissance du post-structuralisme et/ ou de la French Theory ${ }^{52}$. Comme la décade de Cerisy, le colloque de Baltimore a contribué à construire une image de groupe de la «nouvelle critique » en dépit des divergences entre les travaux qui lui étaient ponctuellement affiliés, et surtout il a durablement activé la réception de certains nouveaux critiques français aux États-Unis. Roland Barthes et son élève Tzvetan Todorov, Lucien Goldmann, Georges Poulet y participent avec des chercheurs d'autres disciplines, notamment Charles Morazé, Jean Hyppolite, Jacques Lacan et Jacques Derrida.

L'évolution de ces colloques internationaux appelle trois remarques. D'abord, elle permet d'observer la manière dont la circulation transnationale des « nouveaux critiques » est pluridisciplinaire et surtout très proche des sciences sociales et de leurs réseaux. Ensuite, elle montre que la circulation transnationale des critiques et du même coup la construction progressive d'un espace transnational des théories
48. Voir Giuseppe Bianco, "Le long et monotone chapelet de l'Esprit universel. Disciplinarisation et internationalisation dans les congrès de philosophie ", Revue de métaphysique et de morale, 84, 2014, p. 483-497 ; Éric Brian, "Transactions statistiques au XIXe siècle. Mouvements internationaux de capitaux symboliques ",
Actes de la recherche en sciences sociales, 145, 2002, p. 34-46 et, Anne Rasmussen, "Les Congrès internationaux liés aux Expositions universelles de Paris (1867-1900) ", Mil neuf cent. Revue d'his toire intellectuelle (Cahiers Georges Sorel), 7, 1989, p. 23-44.

49. Georges Poulet et Jean Ricardou (dir.),
Les Chemins actuels de la critique, Paris Plon, 1967

50. Serge Doubrovsky, "Pourquoi la Nouvelle Critique ». Critique et objectivité, Paris, Mercure de France, 1967.

51. Richard Macksey et Eugenio Donato (dir.), The Structuralist Controversy. The Languages of Criticism and the Sciences of
Man, Baltimore, Johns Hopkins University Press, 1970.

52. Voir François Cusset, French Theory. Foucault, Derrida, Deleuze \& Cie et les mutations de la vie intellectuelle aux États-Unis, Paris, La Découverte, 2003. 


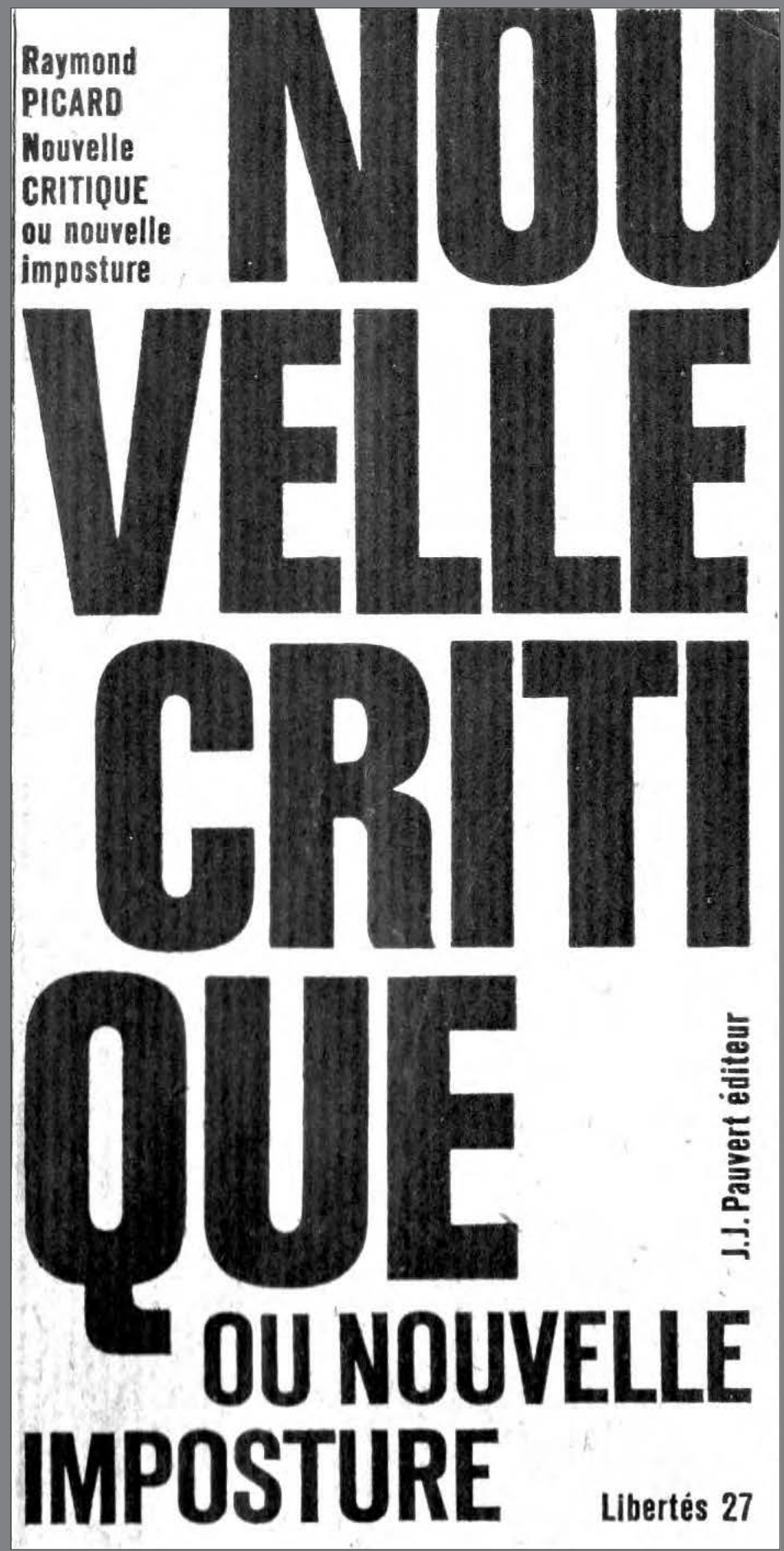

RAYMOND PICARD, Nouvelle Critique ou nouvelle imposture ?, 1965. 


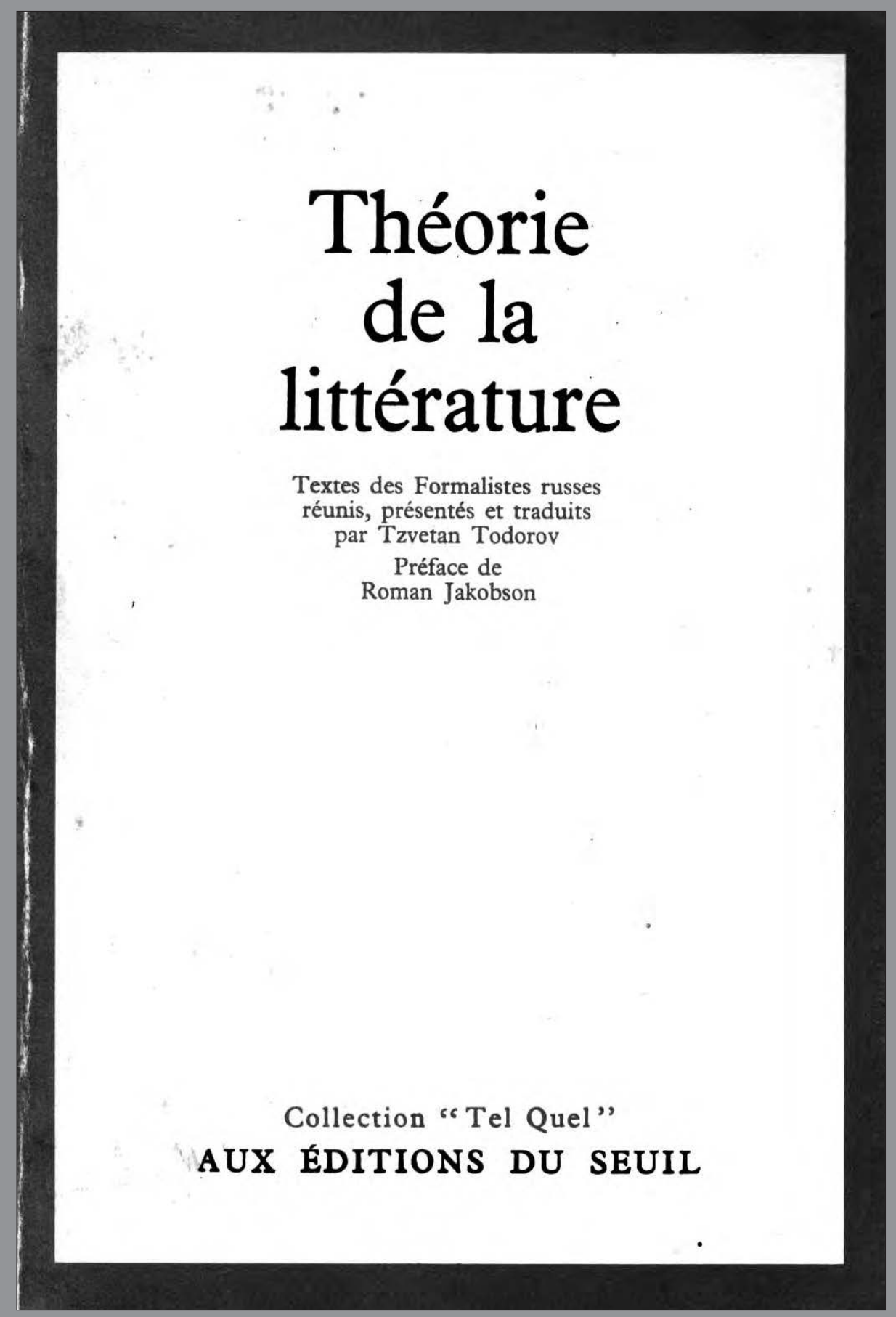

DE GAUCHE À DROITE : Tzvetan Todorov (dir.), Théorie de la littérature. Textes des Formalistes russes réunis, 1965. Robert Emmet Jones, Panorama de la nouvelle critique en France. De Gaston Bachelard à Jean-Paul Weber, 1968. Revue Poétique, 1, 1970. 


\section{Poétique}

revue de théorie et d'analyse littéraires

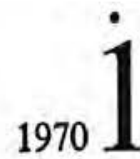

Présentation 1

Roland Barthes, Par où commencer? 3

Jean-Pierre Richard, Balzac, de la force à la forme 10

Harald Weinrich, Structures narratives du mythe 25

Hélène Cixous, L'Écriture comme placement (Henry James) 35

Philippe Lacoue-Labarthe, La fable (littérature et philosophie) 51

Christiane Veschambre, Sur les "Impressions d'Afrique" 64

Mise au point

Hans-Robert Jauss, Littérature médiévale et théorie des genres 79

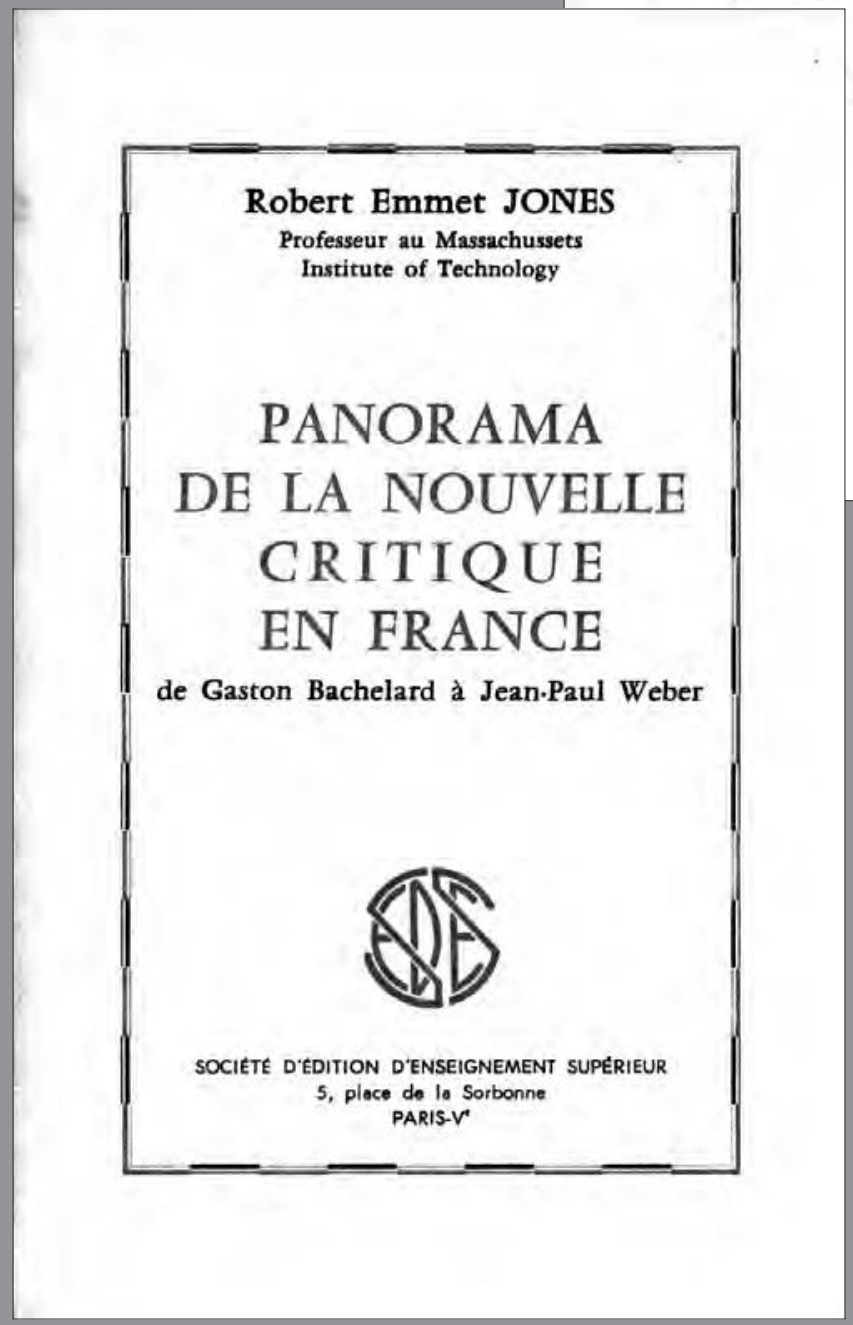

Document

Tzvetan Todorov, Le nombre, la lettre, le mot 102

Vélimir Khlebnikov, Livre des préceptes 112

\section{Seuil}


littéraires n'attendent pas la réception américaine des critiques ni l'élaboration de la French Theory, mais au contraire elles préexistent à cette réception sous différentes formes, individuelles et collectives. De même, dès le départ, et bien que le rôle des participants anglophones et/ou de ceux ayant un capital social et/ou des ressources matérielles au RoyaumeUni ou aux États-Unis soit important, on voit que la construction de l'espace transnational des théories ne se limite pas à des échanges transatlantiques et repose au moins autant sur des trajectoires individuelles transnationales que sur l'internationalisation de certains espaces académiques.

Au colloque de Baltimore, Lucien Goldmann et Tzvetan Todorov, tous deux respectivement émigrés roumain et bulgare et importateurs de György Lukács et des formalistes russes, sont avec Roland Barthes les trois autres représentants de la "nouvelle critique ». Tous trois enseignent à la $6^{\mathrm{e}}$ section de l'EPHE à Paris, dédiée aux sciences sociales. Dans la « nouvelle critique » naissante, ils forment le noyau durable d'un second groupe de producteurs théoriques basés à Paris. Leurs trajectoires se distinguent de celles des représentants de l'École de Genève : marquées par des ruptures biographiques (exil, maladie) et/ou une socialisation plus irrégulière aux instances académiques, elles dessinent des carrières beaucoup moins linéaires et dont le caractère transnational ne se traduit pas immédiatement par l'obtention d'une position stable à l'étranger.

Lucien Goldmann a été élu directeur d'études à la $6^{\mathrm{e}}$ section en 1959 , où il est le premier à consacrer explicitement une partie de son séminaire à des matériaux littéraires (« Sociologie de la philosophie et de la littérature »). Né en 1913 à Bucarest, où il avait obtenu une licence en droit (1934), il avait fui la Roumanie une première fois en direction de Vienne (1930-1931), avant de se rendre à Paris au milieu des années 1930 pour échapper aux persécutions dont les Juifs faisaient l'objet. Suite au décès précoce de son père, juriste, c'est sa mère qui élève Lucien Goldmann dans des conditions matérielles difficiles. À Paris, Lucien Goldmann étudie le droit et l'économie politique. Pendant la guerre il fuit dans le sud de la France, puis en Suisse où il est enfermé dans un camp à Zurich. Il rencontre ensuite Jean Piaget, auprès duquel il élabore son «structuralisme génétique » sur la base d'une alliance de l'épistémologie génétique de Jean Piaget et d'un marxisme anti-stalinien dans lequel György Lukács occupe une place prépondérante. Il entre au CNRS en 1945 et se rapproche du groupe de sociologie de la connaissance de la Sorbonne. Lucien Goldmann publie Le Dieu caché en 1954, qui lui vaut l'admiration de Roland Barthes, de nombreux spécialistes du XVII siècle, et élargit sa réception au-delà des études littéraires et de la philosophie, notamment en sociologie. Il récuse son association à la "nouvelle critique » tout en prenant la défense de Roland Barthes et en s'y alliant ponctuellement contre les attaques dont elle fait l'objet. Ses travaux se concentrent sur l'étude des objets culturels et intellectuels au moyen d'une sociologie de la littérature et de la culture qui utilise la notion de «vision du monde ». Il meurt en $1970^{53}$.

Roland Barthes est de deux ans son cadet. Né en 1915 à Cherbourg, très tôt pupille de la nation suite au décès de son père, il est titulaire d'un DES de lettres obtenu en 1939 à la Sorbonne. Souffrant de problèmes de santé, il ne participe pas à la Seconde Guerre mondiale - comme Lucien Goldmann, réformé pour problèmes de santé - puis renonce à poursuivre ses études et s'insère progressivement dans les milieux littéraires et intellectuels parisiens, en gagnant sa vie par l'enseignement, la publication d'articles critiques, les missions de diplomatie culturelle. Sa carrière se stabilise en 1962 lors de son élection à la $6^{\mathrm{e}}$ section. Il est alors connu comme critique théâtral et littéraire, et auprès d'un public plus large par ses Mythologies, publiées dans les Lettres nouvelles puis en volume en 1957. Roland Barthes passe très tôt beaucoup de temps à l'étranger : à Bucarest et à Alexandrie (1948-1950) dans le cadre professionnel. Dans les années 1960, il fait de nombreux voyages ${ }^{54}$, en Europe, en Afrique du Nord puis en Asie dans les années 1970, lorsqu'il se rend par exemple en Chine avec des membres de la revue Tel Quel. Après la publication du Degré zéro de l'écriture en 1953, Roland Barthes avait été invité dans plusieurs universités au Royaume-Uni, et c'est à la fin des années 1950 qu'il s'était rendu pour la première fois aux États-Unis. Bien qu'il n’y ait jamais passé de long séjour, il y a enseigné en plusieurs endroits et construit des liens solides avec des intermédiaires de la circulation transatlantique des productions intellectuelles pendant cette période, parmi lesquels notamment Susan Sontag ${ }^{55}$. Il est élu au Collège de France en 1977 sur une chaire de "Sémiologie littéraire ", poste qu'il conserve jusqu'à son décès en 1980.

Les nouveaux critiques rattachés à l'École de Genève ou ceux issus de la $6^{\mathrm{e}}$ section possèdent ou accumulent, relativement tôt dans leurs carrières, certaines ressources et dispositions à l'international comme le bilinguisme, qui sont généralement le privilège des dominants dans les pays dominés ${ }^{56}$. Pour Roland
53. Voir Mitchell Cohen, The Wager of Lucien Goldmann. Tragedy, Dialectics, and a Hidden God, Princeton (NJ), Princeton University Press, 1994.

54. T. Samoyault, op. cit., p. 370 sq.
55. Sur la réception de Roland Barthes aux États-Unis, on se permet de renvoyer à : Lucile Dumont, "The moving frontiers of intellectual work. The importation and early reception of Roland Barthes' works in the
United States (1960s-1980s)", Sociologica, 1, 2017 (en ligne).

56. Voir par exemple Gisèle Sapiro, "Le champ est-il national ?... ", art. cit., et AnneCatherine Wagner, «Le jeu de la mobilite et de l'autochtonie au sein des classes supérieures ", Regards sociologiques, 40, 2010, p. 89-98. 
Barthes et Gérard Genette, qui ne parlent pas ou peu de langues étrangères, l'inscription et l'investissement dans les processus de circulation internationale autorisent la connaissance de plusieurs espaces nationaux et surtout l'insertion dans des réseaux institutionnels internationaux (culturels, éducatifs, intellectuels). Ils appartiennent en outre à des institutions qui promeuvent et soutiennent la circulation internationale de leurs membres et constituent des espaces internationalisés du champ académique. Ainsi, la $6^{\mathrm{e}}$ section de l'EPHE se distingue des facultés de lettres, tournées vers les espaces nationaux, par son inscription dans un espace et des réseaux transnationaux. Son lien aux fondations américaines, à l'École libre des hautes études créée auparavant à New York ${ }^{57}$, de même que son soutien à la circulation internationale des chercheurs et à la connaissance de domaines étrangers ${ }^{58}$ inscrivent l'établissement dès sa création en 1947 dans des espaces académiques internationalisés.

\section{La poétique et les usages stratégiques de l'international}

Le dépassement des hiérarchies symboliques entre plusieurs espaces nationaux repose en partie sur des usages stratégiques de l'international. Ceux-ci peuvent être saisis comme "une relation inconsciente entre un habitus et un champ ${ }^{59}$ » qui met à profit des dispositions permettant une implication dans la circulation des idées et du même coup la prise et/ou le maintien d'une position dans le champ académique tant au niveau national qu'au niveau international. Deux phénomènes concomitants permettent de saisir ces usages : premièrement, le travail d'intermédiation que supposent « l'import-export intellectuel $»^{60}$ et plus amplement la mise en circulation des idées, des théories et des textes; deuxièmement, le projet d'élaboration de théories «sans frontières » rendu possible par ce travail d'intermédiation et en même temps dépendant de lui.

En 1965, au moment de la querelle avec Raymond Picard, Roland Barthes tente de constituer la sémiologie comme science et comme discipline, en partie sur la base de l'importation de références étrangères dans l'espace académique français. Il élabore un projet de collection intitulé " bibliothèque de sémiologie ${ }^{61}$, soulignant la nécessité de « fournir au public cultivé de langue française (professeurs, étudiants, chercheurs, intellectuels de toutes disciplines) $»^{62}$ les développements récents du savoir linguistique et sémiologique. La traduction s'impose comme moyen de construire un corpus international. Les langues sources privilégiées sont l'anglais et le russe. Ce dernier représente pour Roland Barthes et pour les promoteurs du structuralisme un important réservoir de travaux de linguistique littéraire et de sémiotique de la culture :

\begin{abstract}
On essaiera de susciter et de rassembler le plus grand nombre possible d'ouvrages originaux en français. Cependant, il faut bien marquer qu'on accordera beaucoup de place aux traductions. C'est une illusion de croire que le public français, même cultivé, lit facilement des livres en anglais, surtout quand ces livres sont en eux-mêmes difficiles, et la sémiolinguistique américaine est riche d'ouvrages notoires mais non traduits, à plus forte raison en ce qui concerne le domaine russe, où les études de linguistique littéraire sont plus développées qu'ailleurs ${ }^{63}$.
\end{abstract}

Parmi les neuf publications incluses dans le projet, quatre sont des traductions. Roland Barthes propose celle d'un ouvrage du folkloriste russe Vladimir Propp, paru en russe en 1928, à traduire du russe mais pour lequel l'anglais, langue dans laquelle Roland Barthes cite significativement le titre de l'ouvrage (Morphology of Folktale) semble jouer le rôle de langue véhiculaire. Il propose ensuite un texte du linguiste américain Noam Chomsky, un recueil d'essais du philosophe américain John Langshaw Austin et un ouvrage du sémioticien bulgare exilé aux États-Unis Thomas Sebeok, tous trois à paraître en anglais. Parmi les ouvrages en français se trouvent deux textes du linguiste Nicolas Ruwet, un recueil de textes de sémioticiens et stoïciens anciens et médiévaux, la

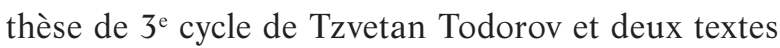
de Roland Barthes (Système de la mode, et son article du dossier consacré à l'analyse structurale des récits dans le numéro 8 de Communications ${ }^{64}$ ). La collection ne voit pas le jour et tous les textes paraissent dans d'autres collections, à l'exception de celui de Thomas Sebeok qui n'est pas publié. Celui de Vladimir Propp sort en 1970 au Seuil, dans la collection « Poétique » rattachée à la revue du même nom. Il est traduit en français par Marguerite Derrida, Tzvetan Todorov et Claude Kahn et ouvre une longue série de travaux consacrés à la narratologie. section de l'EPHE et les études sur l'espace russe, soviétique et est-européen ", Actes de la recherche en sciences sociales, 210 , 2015, p. 60-81.

59. Pierre Bourdieu, Questions de sociologie, Paris, Minuit, 1980, p. 119.
60. P. Bourdieu, "Les conditions sociales de la circulation internationale des idées ", art. cit., p. 3.

61. "Projet d'une bibliothèque de sémiologie présenté par Roland Barthes ", daté du 23 novembre 1965, Seuil.
62. Ibid.

63. Ibid.

64. Voir le numéro de la revue Communications, 8, 1966 : "Recherches sémiologiques : l'analyse structurale du récit ». 
Tzvetan Todorov (1939-2017), né en Bulgarie, élève de Roland Barthes à la $6^{\mathrm{e}}$ section et spécialiste de littérature, incarne de façon paradigmatique la figure de l'importateur parmi les promoteurs des approches théoriques de la littérature. Sa trajectoire biographique est marquée par l'exil et par l'acquisition d'une position durable d'intermédiaire. Arrivé en France au début des années 1960, Tzvetan Todorov a fait la totalité de sa carrière au CNRS, où il est entré en 1968. Il a raconté à plusieurs reprises comment l'approche théorique de la littérature se présentait initialement pour lui comme une manière de contourner les analyses imposées par le régime soviétique dans les études littéraires bulgares, dominées par la philologie ${ }^{65}$. Sa volonté de contourner les assignations à des espaces et des identités nationales marginalisées dans les espaces académiques apparaît dans son récit comme un des moteurs principaux de son engagement dans la production théorique en littérature. Il a ainsi également rapporté que lors de son arrivée en France, il a d'abord été orienté vers un département de littérature slave par le doyen de la Faculté des lettres de Paris. Avec un regard rétrospectif qui a certainement rigidifié le souvenir de cet épisode, il a confié : «Je m'intéressais à la littérature en soi, la théorie littéraire, la stylistique générale. (Le doyen) m'a regardé comme un extraterrestre et m'a dit : Cela n'existe pas, la littérature doit être étudiée dans une perspective historique et nationale. [...] essayez les littératures slaves, nous avons de très bons spécialistes dans ces disciplines ${ }^{66}$ ! » Après un rendez-vous avec une spécialiste de littérature russe qui ne débouche sur rien d'assez convaincant pour le faire rester dans ce domaine - « je ne voulais pas écrire une étude sur Blok, je n'étais pas venu en France pour cela ${ }^{67}$ !»-, il rencontre finalement Gérard Genette qui le dirige vers le séminaire de Roland Barthes.

$\mathrm{Ce}$ « désir de France » sur lequel revient également longuement Julia Kristeva ${ }^{68}$, elle aussi originaire de Bulgarie et arrivée à Paris au début des années 1960, ne prend pas pour ces jeunes chercheurs exilés la forme d'une référence à la littérature française ni aux définitions strictement nationales de la littérature mais celle d'une référence à la littérature comme valeur partagée et universelle. En effet, Julia Kristeva, scolarisée au lycée russe de Sofia et qui maîtrise plusieurs langues, avait introduit en France les travaux du théoricien russe de la littérature Mikhaïl Bakhtine. Quelques années plus tôt, Lucien Goldmann avait quant à lui introduit les travaux de György Lukács en français. L'arrachement progressif de Roman Jakobson aux "études slaves » aux États-Unis et son cheminement vers la linguistique et la poétique générales illustrent des mécanismes similaires basés sur un éloignement progresif de la référence au local et/ou aux espaces nationaux d'origine. Toutefois, on ne devient pas importateur seulement du fait de l'exil, de même qu'on n'abolit pas ses origines dans des aspirations à l'universel littéraire uniquement du fait de ses intérêts intellectuels. Les origines sociales privilégiées de Tzvetan Todorov, dont le père était diplômé de lettres et de philologie, directeur de la Bibliothèque nationale de Sofia puis enseignant à l'université ; de Julia Kristeva, fille d'un père médecin et initiée tôt à la culture artistique et lettrée ; et de Roman Jakobson, issu d'une famille d'industriels russes, ont sans conteste nourri leurs aspirations au théorique et à la généralisation et favorisé cette internationalisation et ces usages stratégiques de l'international. Il est vrai, cependant, qu'en tant que « méridien de Greenwich littéraire » ${ }^{69}$, Paris joue un rôle spécifique, à la fois littéraire et politique, dans la construction d'un espace des théories littéraires relativement autonome des frontières nationales. Comme l'a montré Pascale Casanova, « la consécration parisienne est un recours nécessaire pour les auteurs internationaux de tous les espaces dominés. [...] La croyance dans l'effet de la capitale des arts est si puissante que [...] elle est devenue le lieu à partir duquel, jugés, critiqués, transmués, les livres et les écrivains peuvent se dénationaliser et devenir ainsi universels $»^{70}$.

C'est en effet notament par le biais de traductions, parfois vers le français, que s'est constitué et que s'est exporté le canon des théories littéraires issues du structuralisme. La parution de Théorie de la littérature, un recueil de textes des formalistes russes, pour la première fois réunis dans un même volume, est le fait de Tzvetan Todorov, qui les rassemble et les traduit. Le recueil parait aux éditions du Seuil en 1965, dans la collection « Tel Quel», prolongement de la revue du même nom et également dirigée par Philippe Sollers, avec une préface de Roman Jakobson. Ce linguiste russe, figure de premier plan pour le structuralisme, avait notamment été l'auteur d'une analyse fondatrice des « Chats » de Charles Baudelaire avec Claude Lévi-Strauss, rencontré à New York ${ }^{71}$. Son installation aux États-Unis pendant la Seconde Guerre mondiale, avant qu'il ne soit en poste à Harvard puis au MIT, l'insère dans plusieurs
65. Entretien avec Tzvetan Todorov, Paris juin 2014.

66. Tzvetan Todorov, Devoirs et délices. Une vie de passeur. Entretiens avec Catherine
Portevin, Paris, Seuil, 2002, p. 73. 67. Ibid., p. 74

68. Julia Kristeva, Je me voyage. Mémoires Entretiens avec Samuel Dock, Paris, Fayard,
2016.

69. Pascale Casanova, La République mondiale des Lettres, Paris, Seuil, 1999.

70. Ibid., p. 190-191.
71. Roman Jakobson et Claude Lévi-Strauss, " "Les Chats" de Charles Baudelaire", L'Homme, 2(1), 1962, p. 5-21 et, Corres pondance, 1942-1982, Paris, Seuil, 2018 
circuits - académiques, philanthropiques - qui participent de la réception précoce de la linguistique structurale dans les espaces anglophones et de l'élaboration d'un récit des origines du structuralisme qui revoit à la hausse le rôle de Roman Jakobson dans son développement $^{72}$. Théorie de la littérature est rapidement traduit en plusieurs langues. Un dispositif juridique contraint sa diffusion et sa traduction, les éditions du Seuil ne pouvant céder que le travail « de recherche, de rassemblement, de classement et de présentation des textes, ainsi que la préface de Roman Jakobson $»^{73}$. Il paraît en italien en $1968^{74}$, en espagnol en $1970^{75}$, puis en portugais en $1978^{76}$ et en Corée en $1981^{77}$. Manquant de compétences en russe pour traduire les textes originaux, la maison d'édition brésilienne Globo $^{78}$ souhaitait initialement traduire le recueil depuis le français. Elle a finalement fait traduire les textes à partir des versions originales et modifié la composition du recueil, en ôtant le patronage de Tzvetan Todorov. Ces traductions attestent de l'usage du français comme l'une des langues intermédiaires pour la diffusion de certains textes des formalistes et pour leur constitution en corpus théorique. Elles montrent également la place importante du recueil dans la structuration d'un espace transnational des théories littéraires. Signe enfin d'une volonté de s'approprier en français les principes formalistes, leur concept central, la « littérarité », soit la caractéristique de ce qui est littéraire, est traduit pour la première fois en français dans Théorie de la littérature, quand d'autres langues lui préfèreront son original russe, literaturnost.

La traduction, la mise en circulation, la sélection, l'assemblage et le «bricolage » des textes, selon le mot de Claude Lévi-Strauss cher à Gérard Genette, sont au principe du projet de théories « sans frontières". En élaborant Théorie de la littérature, dont la réception internationale s'inscrit dans le contexte de la fin du jdanovisme ${ }^{79}$, Tzvetan Todorov littérarise les formalistes en sélectionnant parmi leurs travaux ceux ayant trait à la littérature. Dans le même temps il les dénationalise en faisant de ce geste la condition de possibilité d'un discours théorique et de son " universalisation apparente $»^{80}$, puisqu'il écarte " toute conclusion qui n'aurait de valeur que pour l'histoire singulière d'une littérature, russe ou autre $»^{81}$ et épure en grande partie les textes de leurs références empiriques ${ }^{82}$. La revue Poétique s'inscrit dans ce prolongement. La collection qui l'accompagne accueille de nombreuses traductions. Celles de l'anglais y occupent à ce jour la plus grande part. Les quatre traductions du russe, qui arrivent en deuxième position - à égalité avec l'allemand sont notoirement faites dans les premières années de son existence ${ }^{85}$. La présentation du premier numéro de la revue s'ouvre sur l'affirmation qu'elle « veut être à la fois le signe et l'un des instruments » d'un « renouveau théorique » des études littéraires qui « implique » l'ouverture « à des recherches antérieures ou parallèles menées hors de France (Formalisme russe, New Criticism anglo-saxon, Literaturwissenschaft allemande) $»^{84}$. Elle prône un décloisonnement de l'analyse des textes littéraires et une rupture avec le nationalisme méthodologique qui impose l'abandon de la littérature comparée, comme méthode et comme horizon de rénovation des études littéraires :

\begin{abstract}
Éditée en France et rédigée en français, Poétique ne sera pas une revue de littérature française. Si l'ouverture théorique implique une rupture décisive avec l'isolationnisme traditionnel de la recherche littéraire en France, elle suppose également une levée des barrières qui divisaient jusqu'ici l'objet même de cette recherche : aucune pensée de la littérature ne peut s'enfermer dans des limites nationales sans s'exposer aux plus graves erreurs de perspective. Aussi, et sans s'arrêter aux remèdes du défunt " comparatisme », Poétique sera-t-elle, dans son objet comme dans sa pratique, une revue sans frontières ${ }^{85}$.
\end{abstract}

Cette "levée des barrières » postulée par la revue et la collection et par certains de leurs auteurs ${ }^{86}$ ne met cependant pas toutes les traditions intellectuelles importées - ni leurs " certificats d'universalité ${ }^{87}$ parfois concurrents - sur le même plan : l'importation des textes en russe d'une part et l'usage du français comme langue intermédiaire pour les traductions suivantes d'autre part montrent l'impératif de traduction
72. Catherine Depretto, "Le formalisme russe et ses sources. Quelques considérations de méthode ", Cahiers du monde russe, 51, 2010, p. 565-579.

73. Lettre de Jacqueline Leeschaeve à Tzvetan Todorov, datée du 21 octobre 1969. Archives de l'IMEC, SEL 3984.8, dossier "Todorov Fichier analyse ".

74. Tzvetan Todorov, I formalisti russi : teoria della letteratura e metodo critico. A cura di Tzvetan Todorov, Turin, Einaudi, 1968. Voir aussi Giuseppina Larocca, "Ancora sulla ricezione della teoria letteraria russa in Italia ", LEA - Lingue e letterature d'Oriente e d'Occidente, 5 , 2016, p. 623-643.

75. Tzvetan Todorov (dir.), Teoría de la literatura de los formalistas rusos, Mexico, Siglo Veintiuno, 1970.

76. Tzvetan Todorov (dir.), Teoria da literatura II. Textos dos formalistas russos. Lisbonne, Ediç̃es 70, 1978.

77. Russia hyeong-sig-ju-eui, Séoul, Ewha Womens University, 1981. Voir par exemple Kim Chie-Sou, "Semiotics in Korea", semioticon.com, 1, 2004 (en ligne).

78. Lettre de Jacqueline Leeschaeve à Tzvetan Todorov, datée du 21 octobre
1969. Archives de l'IMEC, SEL 3984.8, À l'épreuve, 2, 2015. dossier "Todorov Fichier analyse ". 79. F. Matonti, "L'anneau de Mœbius... », p.1.

art. cit.

80. Voir Pierre Bourdieu et Loïc Wacquant, $\quad \mathbf{8 6}$. Voir par exemple René Wellek et Aus"Sur les ruses de la raison impérialiste", tin Warren, La Théorie littéraire, Paris, Actes de la recherche en sciences sociales, Seuil, 1971. 121-122, 1998, p. 109-118.

81. Tzvetan Todorov, "Présentation", in Tzvetan Todorov (dir.), Théorie de la littérature, Paris, Seuil, 1965, p. 24. 82. Ibid.

83. Voir également Fanny Lorent, "La collection "Poétique" : terre d'accueil ",
84. "Présentation ", Poétique, 1, 1970,

85. Ibid.

tin Warren, La Théorie littéraire, Paris,

87. Romain Lecler, " Nouvelles vagues. Le marché-festival de Cannes ou la fabrique française d'un universel cinématographique ", Actes de la recherche en sciences sociales, 206-207, 2015, p. 14-33. 
des langues rares et les modalités similaires de réception pour des langues semi-périphériques (italien, espagnol). Dans le même temps, ces logiques circulatoires montrent que la langue qui devient dominante sur le marché académique international, l'anglais, dispose de ses propres circuits d'importation, renforcés par la présence aux États-Unis d'autres théoriciens émigrés. L'espace transnational des théories littéraires est donc bien polycentrique : plusieurs pôles d'importexport et de diffusion coexistent et interagissent en son sein. C'est d'ailleurs en anglais que Tzvetan Todorov dit avoir en premier connu les formalistes, dans le recueil de Victor Erlich, slaviste d'origine russe émigré aux États-Unis où il obtient un poste à Yale ${ }^{88}$. Et c'est en anglais également que Tzvetan Todorov publie, au début des années 1980, une anthologie de théorie littéraire française dans laquelle il rassemble des auteurs issus de la «nouvelle critique » et de Poétique ${ }^{89}$. Cependant, au-delà de ce que les usages des langues et les circuits d'importation peuvent révéler des hiérarchies symboliques et de leurs investissements stratégiques par les théoriciens, l'espace transnational des théories littéraires se déploie également sur le plan institutionnel, comme le montrent le rassemblement de théoriciens en associations et l'ancrage des théoriciens de la génération Poétique dans les espaces anglophones, plus particulièrement aux États-Unis.

\section{Modalités de contournement des structures disciplinaires nationales: colloques, revues, associations internationales}

Si les promoteurs des approches théoriques de la littérature participent activement du renouveau de la critique et de ses formes académiques, les études littéraires universitaires françaises ne constituent pas leur point de chute privilégié, et non uniquement du fait de leur opposition. L'agrégation de lettres modernes, voie royale pour la consécration académique de cette discipline et la formation d'un corps professoral dédié, n'est créée qu'en 1959. C'est dans ce contexte que les promoteurs des théories littéraires, tant ceux de la première que de la deuxième génération, quoique formés dans les universités (Roland Barthes, Gérard Genette) ou s'en étant rapprochés en premier lieu (Tzvetan Todorov, Julia Kristeva) évoluent pour la plupart hors des études littéraires universitaires. Le choix relativement libre des thématiques d'enseignement et la souplesse générale des emplois du temps au CNRS ou à la $6^{\mathrm{e}}$ section, parfois combinés à l'absence d'emploi académique stable, expliquent en partie la disponibilité des théoriciens pour les nombreux séjours à l'étranger. L'ouverture de nouveaux espaces académiques après 1968, à commencer par le Centre universitaire expérimental de Vincennes où Hélène Cixous, Gérard Genette et Tzvetan Todorov participent à la création du département de «Littérature générale », modifie les équilibres en accueillant les nouvelles approches théoriques de la littérature. Ainsi, au moment où il devient possible de faire carrière dans une filière universitaire qui accueillerait les théories littéraires, la plupart des théoriciens ont déjà bien entamé leur carrière dans d'autres disciplines ou dans d'autres espaces. En France, les transformations de l'enseignement supérieur permettent aux théories littéraires d'entrer dans les circuits universitaires. Aux États-Unis comme au niveau international, c'est souvent par d'autres disciplines que les études littéraires s'institutionnalisent, se structurent et se dotent de lieux transnationaux de régulation et de visibilité. La sémiotique, contrairement aux études littéraires et/ou de langues, permet aux promoteurs des théories de contourner les instances de régulation des disciplines littéraires, pour mieux rejoindre ces dernières «par le haut ». L'association internationale de sémiotique (AIS/International Association for Semiotic Studies), créée en 1969 à Paris, participe de ces instances. Dirigée jusqu'en 1972 par le linguiste français né en Syrie Émile Benveniste, puis par le sémioticien italien Cesare Segre, l'un des introducteurs principaux du structuralisme en Italie, suivi par le philosophe et sémioticien polonais Jerzy Pelc. Elle a compté parmi ses membres une grande partie des promoteurs les plus connus des théories littéraires ou certains de leurs alliés de la première heure, comme Roland Barthes, Julia Kristeva, le linguiste André Martinet ou le critique littéraire et sémioticien italien Umberto Eco.

La création de l'association a été préparée par plusieurs colloques dans les années 1960, ainsi que par les réseaux intellectuels articulés autour de la diffusion $\mathrm{du}$ formalisme et du structuralisme. Un colloque organisé en 1962 à Moscou, "Symposium on the structural studies of sign systems », a notamment permis d'amorcer la réunion des cercles linguistiques de Moscou et de Tartu et nourri le projet de création d'un institut de sémiotique qui aurait servi au développement de la linguistique structurale ${ }^{90}$. Si le contrôle politique soviétique empêche les principales figures de ces groupes de travail - dont Youri Lotman, qui prend la tête au milieu des années 1960 de l'école sémiotique
88. T. Todorov, Devoirs et délices... op. cit., p. 77. 89. Tzvetan Todorov (éd.), French Lite-

Theory Today. A Reader, New York/ Paris, Cambridge University Press/Éd. de la MSH, 1982.
90. Voir Marina Grishakova et Silvi Salupere (éds), Theoretical Schools and Circles in the Twentieth-Century Humanities.
Literary Theory, History, Philosophy, Londres, Routledge, 2015. 
Les membres du comité d'organisation de l'association internationale de sémiotique*

\begin{tabular}{|c|c|}
\hline Pays représentés & Membres du comité \\
\hline $\begin{array}{c}\text { Allemagne } \\
\text { (République démocratique) }\end{array}$ & $\begin{array}{l}\text { Manfred Bierwisch } \\
\text { Wolfgang Sternitz }\end{array}$ \\
\hline Allemagne (République fédérale) & $\begin{array}{l}\text { Hansjakob Seiler } \\
\text { Harald Weinrich }\end{array}$ \\
\hline Danemark & Holger Sorensen \\
\hline États-Unis & $\begin{array}{c}\text { Roman Jakobson } \\
\text { Michael Shapiro } \\
\text { Thomas Albert Sebeok }\end{array}$ \\
\hline France & $\begin{array}{l}\text { Roland Barthes } \\
\text { Émile Benveniste } \\
\text { Algirdas Julien Greimas } \\
\text { Claude Lévi-Strauss }\end{array}$ \\
\hline Grande-Bretagne & $\begin{array}{l}\text { Donald M. Mackay } \\
\text { Ernst Hans Gombrich }\end{array}$ \\
\hline Hongrie & Iván Fónagy \\
\hline Italie & $\begin{array}{l}\text { S. Avallo } \\
\text { Aldo Rossi }\end{array}$ \\
\hline Pologne & $\begin{array}{c}\text { Jerzy Kuryłowicz } \\
\text { Maria Renta Mayenowa } \\
\text { Stefan Żółkiewski }\end{array}$ \\
\hline Roumanie & Mihai Popp \\
\hline Suède & Hans Regnell \\
\hline Tchécoslovaquie & Jiří Levý \\
\hline Union soviétique & $\begin{array}{c}\text { Tamaz V. Gamkrelidez } \\
\text { Vyačeslav V. Ivanov } \\
\text { Youri M. Lotman } \\
\text { Sebastian K. Shaumyan }\end{array}$ \\
\hline
\end{tabular}

* "Constitution de l'Association internationale de sémiotique. Procès-verbal de deux réunions d'organisation ", Social Science Information, 2-3(6), 1967, p. 55-57 et en particulier p. 56 
de Tartu et de Moscou ${ }^{91}$ - de se déplacer, ils sont rejoints à deux reprises à l'occasion de summer schools par Roman Jakobson et Thomas Sebeok, qui assurent la connexion entre les États-Unis et les espaces académiques et intellectuels du monde soviétique ${ }^{92}$. Une autre conférence internationale de sémiotique organisée en 1966 à Kazimierz nad Wisłā en Pologne ${ }^{93}$ avait été à l'origine de la création d'une première association internationale de sémiotique qui n'avait pu réaliser ses premiers objectifs. Ces objectifs étaient multiples : " réunir les chercheurs concernés par la sémiotique [...] Promouvoir les recherches dans ce domaine, grâce à la coopération et aux contacts internationaux [...] Permettre l'organisation systématique de réunions de travai ${ }^{94} »$. L'association entendait également rechercher « l'appui matériel et moral des organisations internationales, en particulier de l'Unesco ${ }^{95} »$. La réunion de création du comité pendant le colloque a rassemblé huit personnes, présentées selon leur pays d'appartenance (France, États-Unis, Allemagne, Danemark, Italie, Pologne, Tchécoslovaquie, Union soviétique). Roman Jakobson y représente les États-Unis, tandis que le linguiste et sémioticien Algirdas Julien Greimas, né en Russie de parents lituaniens, qui avait effectué ses études et le début de sa carrière entre la Lituanie et la France avant d'être élu directeur d'études à la $6^{e}$ section en 1965, représente la France. Tous deux jouent à la fois le rôle de représentants nationaux et d'intermédiaires transnationaux. L'association décide de la création d'un comité d'organisation dont les personnes, assure-t-elle, ont été choisies pour « leur apport scientifique personnel, mais aussi (d'après) la nécessité d'équilibrer les représentations nationales ${ }^{96}$. Le pays d'exercice des spécialistes prime sur le pays d'origine, et l'association est investie Paul de Manière très inégale par les différents pays, la France, l'Union soviétique et les États-Unis étant de nouveau les pays les plus représentés. Le groupe qui représente la France est composé de quatre figures à la renommée internationale et fondatrices pour le structuralisme [voir tableau, p. 61].

Comme cela a pu être montré sur des associations européennes de sciences sociales ${ }^{97}$, la réflexion sur l'organisation internationale n'oblitère pas totalement la question des logiques nationales, puisqu'il est décidé que les membres du comité d'organisation « se chargent de créer, dans leurs pays respectifs, des associations sémiotiques nationales, ou des groupes similaires $»^{98}$. Enfin, le comité décide de l'organisation d'un congrès en 1967, qui « devra représenter tous les organismes nationaux et toutes les tendances actuelles de la sémiotique $»^{99}$. Un sous-groupe composé de Algirdas Julien Greimas, Youri Lotman, Thomas Sebeok et Wojciech Skalmowski se charge d'étudier les possibilités de créer une revue ou de s'associer à une revue existante. C'est chose faite assez rapidement puisque Studies in Semiotics/Recherches sémiotiques paraît entre janvier 1967 et décembre 1968 dans le cadre d'une publication de l'Unesco, Social Science Information/Information sur les sciences sociales, avec Algirdas Julien Greimas puis Thomas Sebeok comme rédacteurs en chef. Deux femmes y sont mentionnées comme rédactrices adjointes, Julia Kristeva et Josette Rey-Debove. Après quelques années, la revue Semiotica devient le nouveau journal officiel de l'association. Soutenue par le Conseil international de la philosophie et des sciences humaines, qui rassemble les associations internationales spécialisées en sciences humaines au sein de l'Unesco à partir de $1949^{100}$ ainsi que par la $6^{\text {e }}$ section de l'EPHE, elle est créée en 1969. Elle aspire à " présenter les recherches des savants de l'Est aussi bien que de l'Ouest dans l'esprit d'un large dialogue » et promeut la sémiotique en tant que «méthodologie des sciences humaines : le levier par lequel s'effectue la " scientifisation" des pratiques sociohistoriques, de même que l'interrogation de cette scientifisation par la théorie (la sociologie, l'histoire) $»^{101}$. Le comité de rédaction de Semiotica, dirigé par Thomas Sebeok, comprend Roland Barthes, Umberto Eco, Youri Lotman, Jerzy Pelc, Michael Shapiro, Hansjakob Seiler et le linguiste belge Nicolas Ruwet. De nouveau, Julia Kristeva et Josette Rey-Debove y sont mentionnées comme rédactrices-adjointes. Julia Kristeva se charge de traiter la correspondance française au Centre d'études des communications de masse (CECMAS) lui-même à la $6^{\mathrm{e}}$ section dont le pôle littéraire, autour de Roland Barthes, fédère les promoteurs des théories littéraires en France.

Aux États-Unis, la Semiotic Society of America (SSA) voit le jour au milieu des années 1970. Thomas Sebeok la préside en 1984. Il inaugure une série de présidences proches des théories littéraires françaises : en 1986, c'est Michael Riffaterre, proche de Poétique, qui préside l'association. Il est suivi par Jonathan Culler en 1988, ancien élève de Harvard et d'Oxford
91. Sign, System, Studies, 1, 1964.

92. Voir Michel Aucouturier, "La science de la littérature : une renaissance ", Revue des études slaves, 57(2), 1985, p. 295-307.

93. Voir Claude Brémond, "La conférence de sémiotique de Kazimierz ", L'Homme, 7(2), 1967, p. 123-125.

94. "Constitution de l'Association internatio- nale de sémiotique. Procès-verbal de deux réunions d'organisation ", Social Science Information, 2-3(6), 1967, p. 55-57. 95. Ibid.

96. Ibid., p. 56.

97. Voir Thibaud Boncourt, "La science internationale comme ressource. Genèse et développement comparés des associa- tions européennes de sciences sociales ", Revue française de sociologie, 57(3), 2016, p. 529-561

98. Ibid., p. 56 .

99. Ibid., p. 56.

100. Voir Chloé Belloc, "La création du

Conseil international de la philosophie et

des sciences humaines : idéal et réalité d'un engagement scientifique et intellectuel 1947-1955 ", Relations internationales, 130, 2007, p. 47-63.

101. N.S., "Une nouvelle revue: Semiotica ", Annales. Économies, sociétés, civilisations, 25(3), 1970, p. 825-826. 
et professeur de littérature à l'Université de Cornell, et par Robert Scholes, ancien élève de Yale et professeur à Brown University, en 1990, tous deux promoteurs de l'analyse structurale en littérature aux États-Unis. Si l'AIS a incarné dans un premier temps un certain volontarisme internationaliste où la pratique de la théorie - sémiotique, culturelle, inspirée du formalisme et du structuralisme - s'élargissait au-delà des objets littéraires, la SSA semble avoir servi de lieu de subversion des canaux traditionnels de légitimation dans les études littéraires. Dans les deux cas, une alliance avec plusieurs disciplines des sciences humaines et sociales, tant sur les plans institutionnels (par exemple dans la $6^{\text {e }}$ section de l'EPHE ou au CNRS) que disciplinaire et méthodologique (linguistique et anthropologie structurales, sémiologie, sémiotique) s'est révélée indispensable pour contourner les instances régulatrices propres aux disciplines littéraires.

Le caractère pluridisciplinaire et international de la généalogie intellectuelle des théories littéraires ainsi que des enjeux, des espaces et des agents qui ont permis leur émergence interdisent de les circonscrire à l'histoire littéraire, intellectuelle et académique française. Les modalités de leur circulation ont permis la construction progressive d'un espace transnational spécifique appuyé sur des réseaux de personnes, d'organisations et d'institutions internationales qui ont en retour permis la pénétration des théories dans plusieurs espaces nationaux. La constitution d'un espace transnational des théories littéraires et leur institutionnalisation se sont ainsi mutuellement renforcées. Les espaces transnationaux, dont la construction résulte de la combinaison de trajectoires migratoires, de politiques académiques et de projets intellectuels, apparaissent ainsi comme des lieux privilégiés de l'élaboration théorique sur la littérature. La possibilité des migrations intellectuelles, l'accueil à l'étranger des populations déplacées et la conversion des ressources internationales dans le travail d'intermédiation en sont des illustrations, dans la mesure où « toute trajectoire d'exil place l'individu dans une position multiple d'intermédiaire entre groupes, espaces sociaux, entre cultures $^{102} »$. Dans cette perspective, la volonté de construire un projet théorique « sans frontières » est bien inséparable de ses conditions sociales de possibilité, et dans le même temps elle repose sur l'arrachement des productions intellectuelles à ces dernières.

Est-ce à dire que seules les productions théoriques circulent à l'étranger, ou sont au principe de la construction d'un espace transnational ? D'autres volets de notre enquête montrent qu'il n'en est rien. Plutôt, les représentants des études littéraires en poste dans les universités françaises circulent par d'autres canaux. Dans les années 1970, à l'heure où les États-Unis adoptent la théorie littéraire française dans de nombreuses institutions d'élite, ces universitaires trouvent certes une forme de concurrence pour la circulation internationale dans la recherche et la critique littéraire qui était jusque-là d'une ampleur moindre. Mais les départements de français outreAtlantique sont loin d'être conquis dans leur ensemble par la théorie et la critique littéraire françaises des années 1960. En effet, sur le plan pédagogique, celleci permet de changer les manières d'enseigner et l'appareil critique qui accompagne les textes. Ceux-ci sont toutefois d'une importance relative dans des départements consacrés en grande partie à l'apprentissage d'une langue et d'une culture étrangère, et plus irrégulièrement (en fonction des étabissements ou des effectifs enseignants et étudiants par exemple) à la formation à la recherche spécialisée. La réception des approches théoriques françaises de la littérature, dès lors, élargit l'éventail méthodologique et thématique d'analyse de la littérature mais ne transforme pas drastiquement le programme des textes littéraires de français, encore moins celle des départements de littérature. Ils continuent à fonctionner sur un découpage chronologique, avec pour mission première d'enseigner la langue française et de diffuser, avec plus ou moins de souplesse sur sa définition, le canon littéraire national. C'est dans ce cadre que ladite « critique universitaire » a elle aussi son espace transnational, également structuré autour d'associations, de colloques et de revues pour l'enseignement du français à l'étranger. Il y a simultanément plusieurs circuits de diffusion des études littéraires françaises, dont l'assemblage dessine des espaces transnationaux qui se croisent sans se superposer totalement. La circulation internationale des biens symboliques, de ce point de vue, doit toujours être envisagée comme se déroulant dans un espace multidimensionnel et stratifié, dans lequel se nichent les circulations d'autres biens symboliques. La polarisation interne à l'espace transnational des théories littéraires ne dit pas autre chose : la récurrence des circulations Est-Ouest, la domination de la triade France/États-Unis/Union soviétique et l'investissement différencié de cet espace par les agents et les institutions, invitent à porter une attention redoublée aux mécanismes de division internationale du travail intellectuel. 